\title{
Stable open-shell aromatic nitric acid radicals: accessing highly efficient photothermal conversion with restricted radiative decay
}

\author{
Zejun Wang, ${ }^{1}$ Jiawen Zhou, ${ }^{1}$ Yiheng Zhang, ${ }^{1}$ Weiya Zhu, ${ }^{1}$ Yuan $\mathrm{Li}^{1,2 *}$
}

1 State Key Laboratory of Luminescent Materials and Devices, Institute of Polymer Optoelectronic Materials and Devices, Guangdong Provincial Key Laboratory of Luminescence from Molecular Aggregates, School of Materials Science and Engineering, South China University of Technology, 381 Wushan Road, Guangzhou 510640, P. R. China.

2 Guangdong Provincial Key Laboratory of Luminescence from Molecular Aggregates, South China University of Technology, Guangzhou, 510640, P. R. China.

KEYWORDS: Radical, organic semiconductor, open-shell, photothermal conversion, electron spin

\begin{abstract}
Most of the open-shell radicals are usually thermodynamically or kinetically unstable in air due to their incompletely satisfied valency. As phenol radical without steric hindrance group protection, aromatic nitric acid radical exhibits high electrochemical and thermal stability due to its rich resonance structures including closed-shell nitro-like and open-shell nitroxide structure with unpaired electrons delocalized in conjugated backbones. Herein, a series of star-shaped aromatic nitric acid radical materials were prepared via facile demethylation and consequent oxidation of their phenolic hydroxyl precursors in air. Interestingly, they exhibit extremely high spin concentration and highly enhanced nonradiative decay, which make them exhibit great potential for photothermal conversion. Among them, TPA-TPA- $0_{6}$ exhibits high photothermal conversion efficiency and negligible photobleaching effect in seawater desalination. Under irradiation of one sunlight, the water evaporation efficiency of TPA-TPA- $\mathrm{O}_{6}$ is recorded as high as $89.41 \%$ and the water evaporation rate is $1.293 \mathrm{~kg} / \mathrm{m}^{2} \mathrm{~h}$, which represent as the top performance in pure organic small molecule photothermal materials.
\end{abstract}

\section{INTRODUCTION}

Open-shell organic radicals have attracted tremendous attention in recent years, as they exhibit potential applications in many fields such as optoelectronics, 1-7 organic magnets, ${ }^{8}$ photothermal, ${ }^{9-13}$ thermoelectric ${ }^{14-18}$ and spintronic materials, $19-22$ due to their unique chemical/physical properties. ${ }^{11,23-24}$ Narrow optical band gap can be relatively readily achieved through reasonable chemical modification and structural control. 25-28 Considering the good biocompatibility of the radical materials, they have attracted widespread attention in the development of photothermal conversion and biologic applications. 9,29-31 Comparing with the other widely reported photothermal conversion materials such as inorganic materials, organic two-dimensional framework and conjugated polymer materials, ${ }^{12-13,32-34,35,36}$ organic small molecules are relatively overlooked by researchers in this field. In details, the disadvantages of them are well recognized as following aspects: 1) Inorganic materials mainly include carbon materials, gold, and palladium metal nanomaterials. These materials usually contain highly precious and nondegradable metal and will produce potential safety issues for human health when applied in vivo; ${ }^{37-38} 2$ ) Two-dimensional framework and conjugated polymers widely suffer from poor synthesis reproducibility. 39-40 Although these materials exhibit good photothermal effect, they still have drawbacks such as high cost, poor solubility and risk of performance decay in practical photothermal applications. The previous organic small molecules represented by porphyrin, cyanine dyes and other dyes might limit their large-scale application due to their disadvantages including cumbersome synthesis procedure, poor photothermal stability. ${ }^{37}$ Compared with the candidates mentioned above, organic small molecule materials show excellent structural definiteness, convenient preparation and will not cause potential heavy metal ion poisoning. $37-38$

Among the small molecules, the open-shell radicals with high photothermal conversion efficiency and thermal/electrochemical stability are rarely reported in previous work. ${ }^{41-42}$ It is very challenging to prepare airstable organic conjugated radicals due to their intrinsic high reactivity with oxygen. ${ }^{43-46}$ The preparation of stable neutral radicals is not only the basis for in-depth study of radical chemistry theory but also the prerequisite for the realization of multifunctional application. ${ }^{44-46}$ Therefore, photothermal materials based on organic stable radicals, bearing their unique physical and optical properties, can be applied in solar-driven water evaporation, photothermal therapy and photothermal catalysis field by converting near-infrared (NIR) light into heat via nonradiative decay. 12-13,35 Based on our previous research on stable radicals, ${ }^{47-}$ 50 we designed and synthesized a series of aromatic nitric acid radicals (ANARs) without steric hindrance group based protecting groups. ${ }^{49}$ Compared with the traditional triarylamine radical cations, these neutral conjugated nitrogen oxide radicals can be readily prepared, purified and exhibit high thermal/electrochemical/light stability. $11,51-53$

In this work, four star-shaped ANAR materials TPA-TPA$\mathrm{O}_{6}$, TPA-TPZ- ${ }_{6}$, TPA-PY- $0_{8}$, and Spiro- $0_{8}$ were synthesized by introducing different cores such as triphenylamine, triazine, pyrene, spiro fluorene and other groups (Figure 1A, $B, C)$. The detailed synthetic scheme and characterization of the products are provided in the supporting information (SI) (Figure S1-S9). 47, 49, 54-55 The radical species and physical properties of the star-shaped ANAR materials were thoroughly studied by cyclic voltammetry (CV), UV-vis-NIR spectroscopy, and electron spin resonance (ESR) spectrum. 


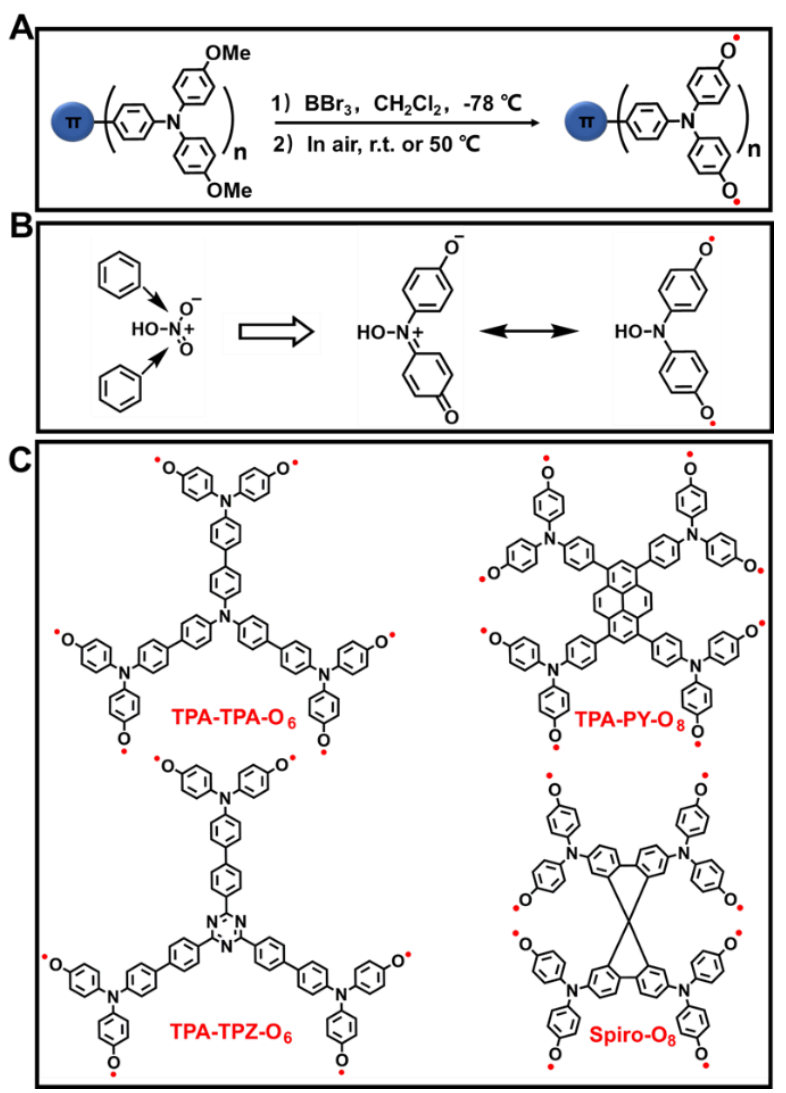

Figure 1. Synthesis procedure and molecular structures. (A) Synthetic routes for the aromatic nitric acid radical materials. (B) Resonance structures of radical motivated from aromatic $\mathrm{HNO}_{3}{ }^{41,49}$ (C) Chemical structure of the four aromatic nitric acid radical materials.

Open-shell ANARs, bearing their unique physical and optical properties were applied in photothermal conversion by converting near-infrared (NIR) light into heat via nonradiative decay. Among them, TPA-TPA- ${ }_{6}$ exhibits the highest photothermal conversion performance compared to other molecules and excellent photo-stability. An interface heating evaporation system based on TPA-TPA-O 6 was established. Under these conditions, high solar-energyto-vapor efficiency of $89.41 \%$ and water evaporation rate of $1.293 \mathrm{~kg} \mathrm{~m}^{-2} \mathrm{~h}^{-1}$ under 1 sun irradiation are obtained. Openshell ANARs enable highly efficient solar energy conversion and provides us a novel strategy for the design of stable organic-small-molecule photothermal materials in solar energy utilization.

\section{RESULTS AND DISCUSSION}

The UV-Vis absorption spectra of TPA-TPA-OMe in the dichloromethane solution and the film are very consistent, with absorption at $305 \mathrm{~nm}$ and $380 \mathrm{~nm}$, respectively (Figure 2A). For the sample TPA-TPA- ${ }_{6}$, the soultion in DMSO exhibited similar absorption spectrum with TPA-TPA-OMe, however, the film absorption of TPA-TPA- ${ }_{6}$ shows a typical and great red shift comparing with that of TPA-TPA-OMe. This clear and long absorption tail in the range of 400-825 $\mathrm{nm}$ Indicates its typical radical character in the solid state, which is widely reported in previous work. ${ }^{11,49}$ In addition, the color of the TPA-TPA- ${ }_{6}$ radical powder is totally black, which is in good agreement with the wide absorption covering the whole visible region. For the pyrene and triazine core, the TPA-PY-OMe and TPA-TPZ-OMe showed the typical wide bandgap absorption spectra with slight redshift comparing with TPA-TPA-OMe. It is noteworthy that both the TPA-PY-O 8 and TPA-TPZ- ${ }_{6}$ exhibited an obvious absorption with edge around $1000 \mathrm{~nm}$ and $800 \mathrm{~nm}$ in film, respectively. (Figure 2B and Figure 2C). The UV absorption of Spiro-OMeTAD and $\mathrm{Spiro}^{-} \mathrm{O}_{8}$ have been
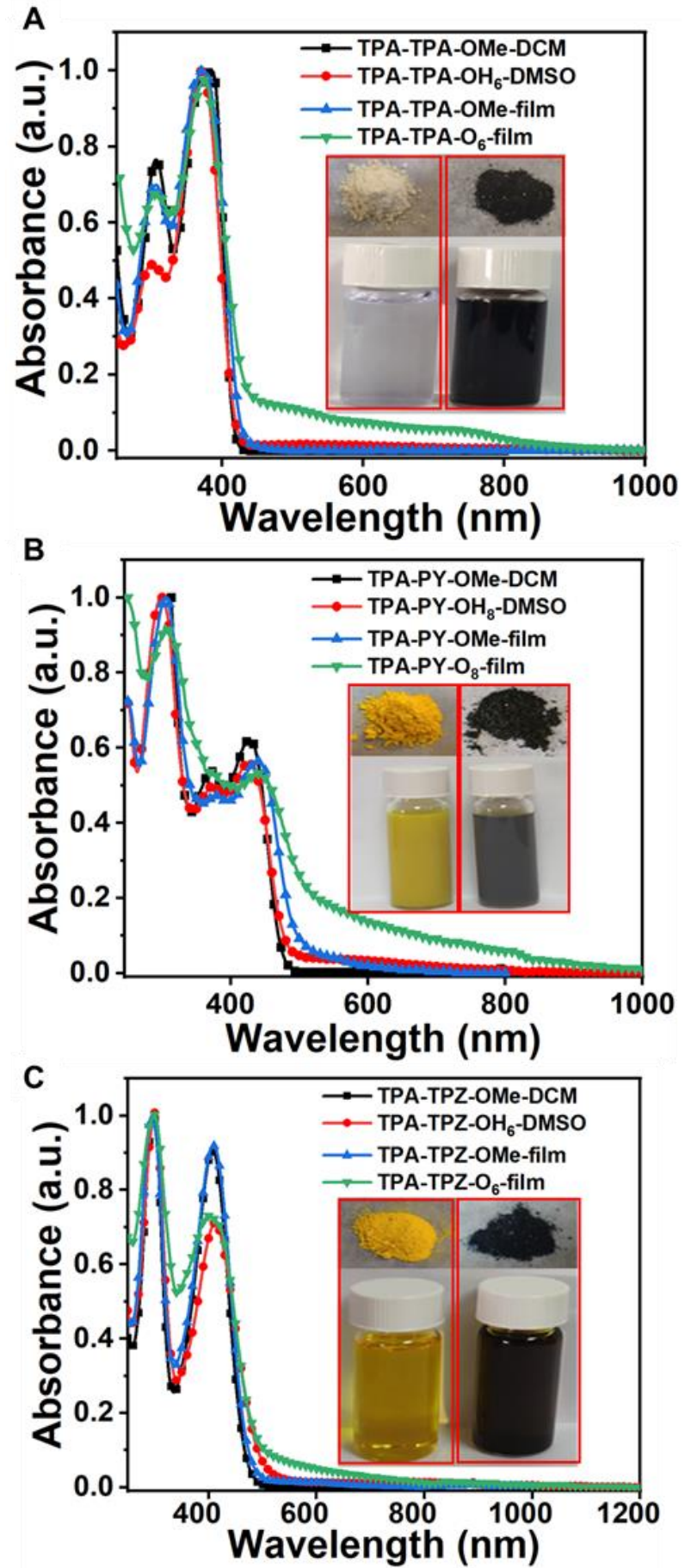

Figure 2. UV-Vis-NIR and PL spectra. (A-C) TPA-TPA-OMe and TPA-TPA-06, TPA-TPZ-OMe and TPA-TPZ-O6, TPA-PY$\mathrm{OMe}$ and $\mathrm{TPA}-\mathrm{PY}-\mathrm{O}_{8}$ in solution as well as film, respectively. 

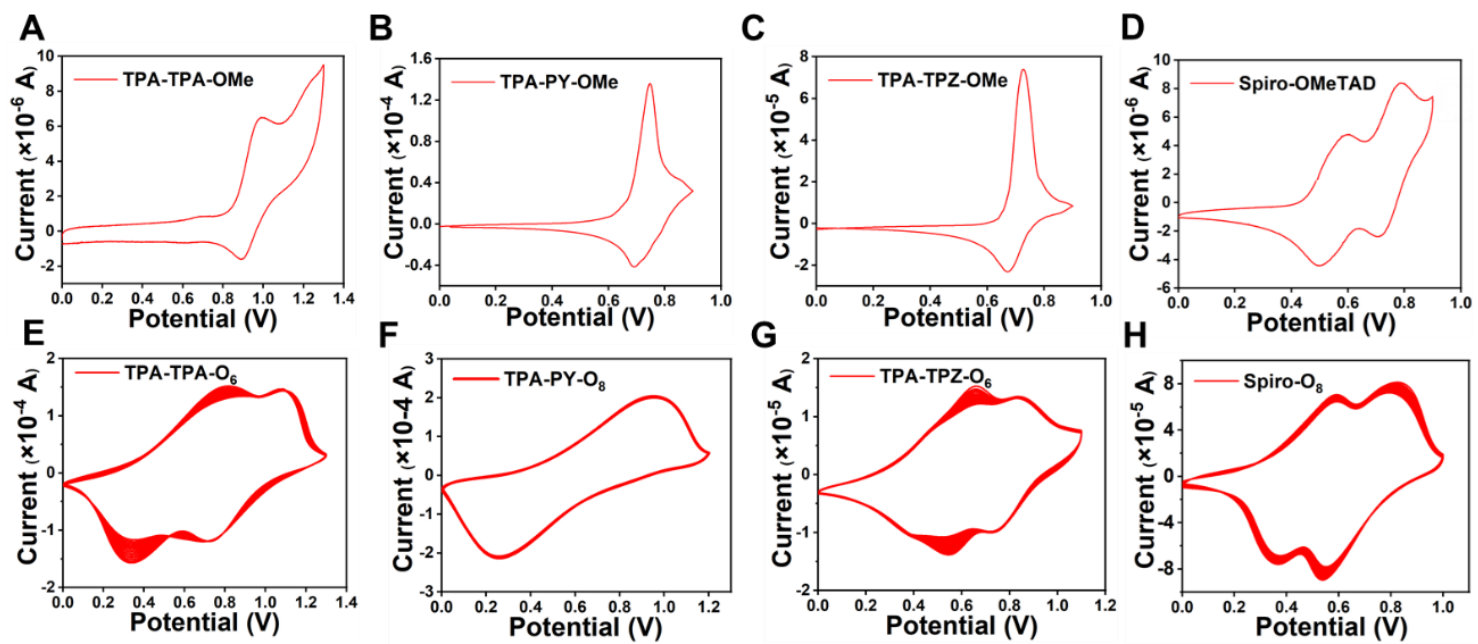

Figure 3. Cyclic voltammograms results. (A-H) TPA-TPA-OMe, TPA-PY-OMe, TPA-TPZ-OMe, Spiro-OMeTAD, TPA-TPA-O 6 , TPA-PY- $0_{8}$, TPA-TPZ- $\mathrm{O}_{6}$ and Spiro- $\mathrm{O}_{8}$, respectively. All of CV curves were measured 15 cycles in dichloromethane solution containing $0.1 \mathrm{M} \mathrm{nBu}_{4} \mathrm{NPF}_{6}$ as a supporting electrolyte and the scan rate was $0.1 \mathrm{~V} / \mathrm{s}$. The methoxy substituted precursors were dissolved in dichloromethane at a concentration of $5 \mathrm{mg} / \mathrm{mL}$ and target radical compounds coated on carbon-glass electrode to form film during measurements. Potential values are reported with saturated calomel electrode as the reference electrode using the $\mathrm{Fc}^{+} / \mathrm{Fc}$ couple $(0.27 \mathrm{~V})$ as an internal standard.

reported in our previous work and they also showed very similar behavior with the other three samples mentioned above. The photoluminescence and photoluminescence quantum yield (PLQY) test of all the precursors of in dichloromethane solution and film showed fluorescent emission between 450 and $570 \mathrm{~nm}$ (Figure 4B). In sharp contrast the moderate PLQY of precursors (Table S1), the corresponding radicals exhibited none of fluorescent emission in film. After demethylation, the PLQYs of these radical products are significantly reduced and all of them showed low fluorescence quantum yields lower than $1 \%$ (Table S1), which indicate the formation of radicals. These results are in good agreement with the previous work in diradicaloids and multiple radicaloids which usually showed obviously lowered PLQY. 56

In order to study the chemical and electrochemical stability, the cyclic voltammetry (CV) test was conducted in film and solution. It can be observed from Figure 3 that all the demethylated counterparts exhibited highly reversible CV curves under the oxidation process and showed negligible change after scanning for 15 cycles (Figure 2). The ANARs showed the excellent reversibility in CV test and this result indicates that these star-shaped ANARs exhibited unexpected electrochemical stability compared to traditional organic radical materials. $26,48,57$ In previous work, the radicaloids widely showed low electrochemical stability. ${ }^{11}$ The HOMO and LUMO energy levels are calculated based on these CV curves and the detailed values are listed in Table S1. The highest occupied molecular orbital (HOMO) and lowest unoccupied molecular orbital (LUMO) energy levels changed slightly to some extent due to the different electron-donating effect while the central cores are modified from triphenylamine to pyrene, triazine and Spiro-fluorene. In addition, the HOMO energy levels of demethylated counterparts TPA-TPA- $0_{6}$, TPA-TPZ- ${ }_{6}$, TPA$\mathrm{PY}_{-} \mathrm{O}_{8}$, and Spiro- $\mathrm{O}_{8}$ are recorded as $-4.90,-4.81,-4.97$, and $4.84 \mathrm{eV}$, respectively. Comparing with the methoxy precursors, the HOMO energy levels of the demethylated radicaloids have been obviously enhanced (Table S1), however, these radicals show comparable electrochemical and air stability with their methoxy precursors.

In order to further disclose the chemical structure of these radicals, the ESR spectrum was applied to characterize the radical spin signal and concentration of these star-shaped molecules. At the same test amount of $0.02 \mathrm{mmol}$, very obvious increase in the relative ESR signal intensity were detected after the demethylation of the corresponding methoxy precursors (Figure 4A and S11), due to the large amounts of radical generation with easy oxidation of phenolic hydroxyl groups in air. ${ }^{49}$ This confirms that star-shaped ANARs molecules showed high concentration of radical spin, which is consistent with the results of ultraviolet absorption spectra and the black color of powder samples. In contrast with the radicals with triazine and pyrene cores with relatively low electrondonating capability, the ESR signal intensity of TPA-TPA-O 6 and Spiro- $_{8}$ are significantly enhanced, which can be understood by the more obvious radical character and lower bandgap. ${ }^{11}$ These results revealed that the strong donor-acceptor conjugation effect would not only reduce the optical bandgap but also determine the paramagnetic property of the radical materials. Besides, the conductivity of all demethylated counterparts has been improved (Figure S10). The triazine and pyrene cores with relatively low electron-donating capability making the HOMO energy levels of TPA-TPZ- ${ }_{6}$ and TPA-PY- ${ }_{8}$ lower than those of TPA-TPA-O 6 and Sprio- $0_{8}$. The lower HOMO energy level makes it difficult to be oxidized by $\mathrm{O}_{2}$ to form radicals, so it shows a weaker signal intensity in the ESR spectrum. In the solid-state, these radical materials showed high spin concentration, and this will lead to highly nonradiative decay and enhanced photothermal conversion efficiency (Figure 4D). 56,58 The PLQY with the value of demethylated counterparts TPA-TPA- ${ }_{6}$, TPA-TPZ- $0_{6}$, TPA-PY-O 8 , and Spiro- $\mathrm{O}_{8}$ are recorded as $0.1 \%, 0.9 \%, 1.0 \%$, and $0.3 \%$ and the PL wavelength is around the corresponding trace 
A

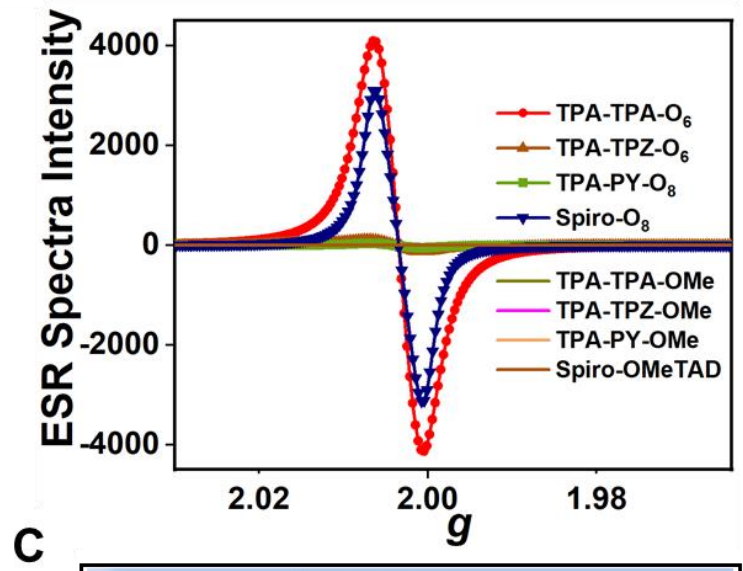

C

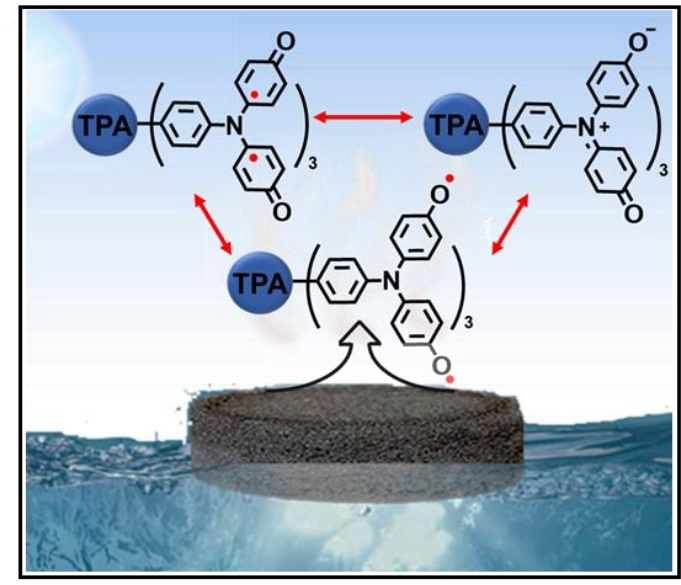

B

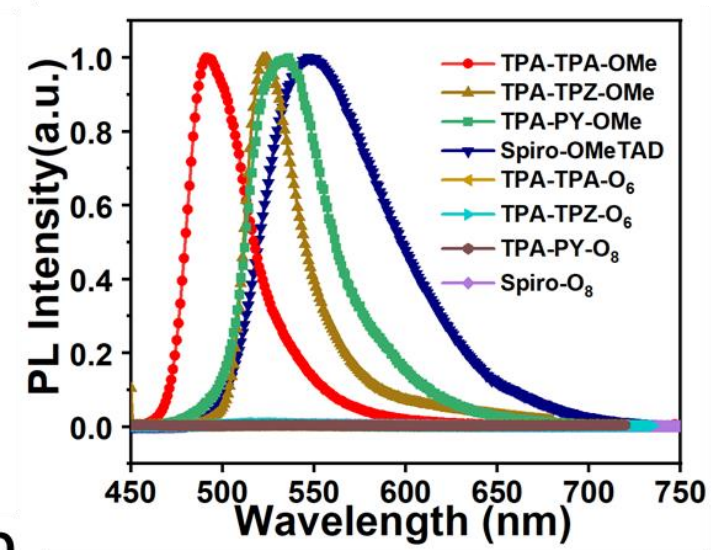

D

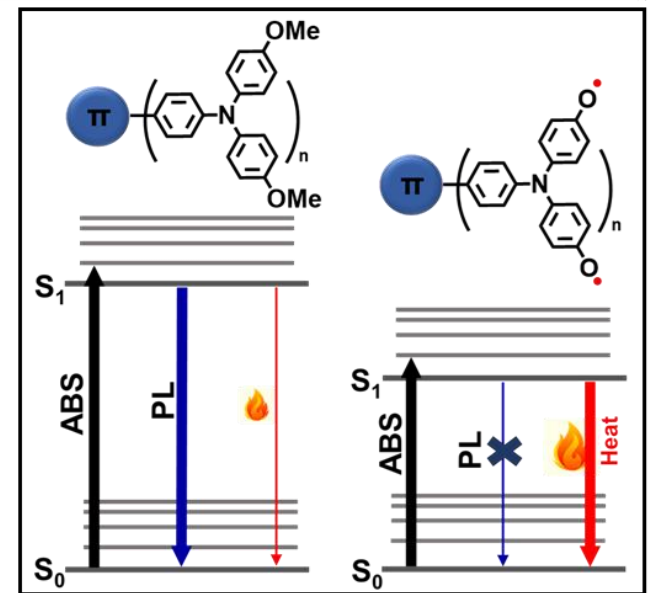

Figure 4. Characterization of aromatic nitric acid radicals. (A) Electron spin resonance spectra of ANARs with the same mole amount $(0.02 \mathrm{mmol})$ of solid samples at room temperature. (B) PL spectra of ANARs before and after demethylation in film, respectively. Film prepared under the same conditions. (C) Schematic illustration of solar-driven water evaporation. (D) The Jablonski diagram illustrates the difference in energy dissipation of excited states before and after demethylation.

protonated phenoxy precursors, respectively. Due to the high concentration of radical spin, the nonradiative decay will be largely boosted, which efficiently enhances the photothermal conversion of TPA-TPA- $\mathrm{O}_{6}$ in the solid state. The sky-blue photoluminescence of demethylated counterpart TPA-TPA- $\mathrm{O}_{6}$ is the most severely quenched in the solid state. The high concentration of radical spin and the almost negligible radiation decay of TPA-TPA- $\mathrm{O}_{6}$ indicate that it will show great application potential in photothermal conversion. 11,12,13, 35

As shown in Figure 6B, TPA-TPA- ${ }_{6}$ show high broadband light absorption. Remarkably, the absorption of TPA$\mathrm{TPA}^{-} \mathrm{O}_{6}$ can be further extended upon aggregation in powder state with the broadened absorption spectrum from 300 to $2000 \mathrm{~nm}$, which strongly facilitates the efficient sunlight harvesting. Comparing with other types of organic photothermal materials, TPA-TPA- $\mathrm{O}_{6}$ exhibit extremely wide absorption spectra. ${ }^{10,12,30,31,59}$

According to the powder absorption spectra, ESR and PL measurements, we propose that the TPA-TPA- $\mathrm{O}_{6}$ might show promising photothermal conversion performance due to the radical-promoted nonradiative decay (Figure 4 and Table S1). ${ }^{48,56}$ The photothermal property of star-shaped radical materials was evaluated by monitoring the temperature increment under irradiation via IR thermal camera, and the detailed test condition is provided in the SI. As shown in Figure 6A, star-shaped radical materials were irradiated by an $808 \mathrm{~nm}$ laser with energy power of $0.8 \mathrm{~W}$ $\mathrm{cm}^{-2}$. After TPA-TPZ- ${ }_{6}$ and TPA-PY-O 8 were irradiated with $808 \mathrm{~nm}$ laser for $60 \mathrm{~s}$, their temperature change from $25{ }^{\circ} \mathrm{C}$ to about $40{ }^{\circ} \mathrm{C}$ and the temperature change slowly. The low concentration of radical spin and the relatively low PLQY value of TPA-TPZ- $\mathrm{O}_{6}$ and TPA-PY- $\mathrm{O}_{8}$ limit their photothermal conversion performance (Figure $4 \mathrm{~A}$ and Table S1). Meanwhile, TPA-TPZ- ${ }_{6}$ and TPA-PY-O ${ }_{8}$ might be partially protonated to form hydroxyl groups, which will also lead to their relatively poor photothermal conversion performance. However, TPA-TPA- ${ }_{6}$ exhibit superior photothermal conversion performance compared to counterparts, for which the temperature increased rapidly from $25{ }^{\circ} \mathrm{C}$ to about $210{ }^{\circ} \mathrm{C}$ within $60 \mathrm{~s}$, revealing a fast photothermal conversion process (Figure $5 \mathrm{~A}$ and $5 \mathrm{C}$ ). ${ }^{10,28 \text {, }}$ 30, 40,42,60-62 As shown in Figure 5B, the temperature change process picture of TPA-TPA- $O_{6}$ powder during the photothermal conversion process under $808 \mathrm{~nm}$ laser for $60 \mathrm{~s}$ was recorded by an IR thermal camera. It was also observed that the temperature of the sample rises regularly with the increase of the power of the laser irradiation intensity (Figure 5C). With the increase of the laser irradiation power to $1.0 \mathrm{~W} \mathrm{~cm}^{-2}$, the temperature of TPATPA $-\mathrm{O}_{6}$ raised up to about $250{ }^{\circ} \mathrm{C}$ within $60 \mathrm{~s}$, and then 
decreased quickly to ambient temperature after turn-off laser. Notably, TPA-TPA-O 6 exhibit excellent stability for ten cycles of laser irradiation on and off (Figure 6C), indicative of outstanding photothermal stability superior to many traditional organic photothermal materials. ${ }^{12}$ Comparing the reported photothermal materials in terms of the temperature increment (Figure $5 \mathrm{C}$ ), it can be found that the TPA-TPA- $\mathrm{O}_{6}$ exhibit a superior photothermal conversion behavior.

Based on the results mentioned above, as a pure organic molecule TPA-TPA- ${ }_{6}$ is a very stable and highperformance photothermal conversion material with an extremely wide and rarely reported absorption spectra in our test condition including powder and dispersed state in PU (Figure 6D). 63-64 Its unique absorption characteristics

make it promising to capture solar energy in solar-driven water evaporation. $65 \mathrm{We}$ employed a commercially available white polyurethane (PU) porous foam as support to establish an efficient interfacial evaporation system by floating on water (Figure 4C). TPA-TPA-O 6 was loaded inside the PU foam by impregnating pure PU foam in solution and drying under $80^{\circ} \mathrm{C}$, obtaining a brownish black PU foam (Figure S16). Among them, the equilibrium temperature of $\mathrm{PU}+\mathrm{TPA}-\mathrm{TPA}-\mathrm{O}_{6}$ foam $(2.0 \mathrm{~cm}$ diameter foam with $30 \mathrm{mg}$ of TPA-TPA-O 6 loading) can reach to as high as $81.2{ }^{\circ} \mathrm{C}$ under $600 \mathrm{~s}$ continuous irradiation (Figure S14), while the PU foam itself only shows a surface temperature of $36^{\circ} \mathrm{C}$ under the same test condition (1
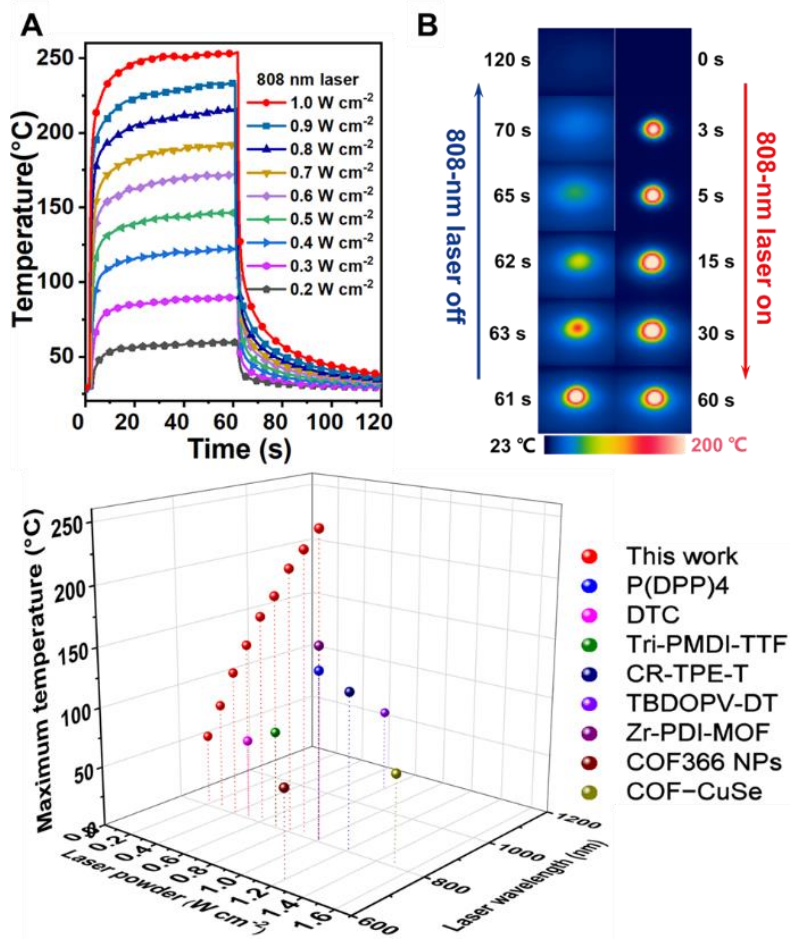

Figure 5. (A) Photothermal conversion behavior of TPATPA-O 6 powder under 808-nm laser irradiation at different laser powers $\left(0.2-1.0 \mathrm{~W} \mathrm{~cm}^{-2}\right)$. (B) IR thermal images of TPA-TPA-O 6 powder (20 mg) under 808-nm laser irradiation (1.0W $\mathrm{cm}^{-2}$ ) and then turned off. (C) The maximum temperatures for reported photothermal materials under laser irradiation. sunlight irradiation, $1.0 \mathrm{KW} \mathrm{cm}^{-2}$ ). As revealed in Figure 6E, the surface temperature of PU+ TPA-TPA-O 6 foam floating on the water is remarkably higher than PU foam under 1 sun irradiation $\left(1 \mathrm{~kW} \mathrm{~m}^{-2}\right)$. The equilibrium temperature of the PU+TPA-TPA- ${ }_{6}$ foam loaded by $30 \mathrm{mg}$ TPA-TPA- ${ }_{6}$ is $45^{\circ} \mathrm{C}$ after 1 sun illumination $\left(1 \mathrm{~kW} \mathrm{~m}^{-2}\right)$ for $1 \mathrm{~h}$ continuous irradiation, which is about $10^{\circ} \mathrm{C}$ higher than that of only PU foam.

The mass change curves of water with PU foam and PU+TPA-TPA- $\mathrm{O}_{6}$ foam was recorded to evaluate the efficiency of solar-driven water evaporation (Figure 6F). It shows an evaporation rate of as high as $1.293 \mathrm{~kg} \mathrm{~m}^{-2} \mathrm{~h}^{-1}$ and the solar-driven water evaporation efficiency $(\eta)$ of $89.41 \%$ under 1 sun illumination (detailed calculation process is shown in Supporting Information). Compared with other types of photothermal conversion materials, this kind of open-shell radicals demonstrate competitive performance of solar thermal conversion among organic materials (Figure S17 and Table S2). 10, 66-68 Comparing with the inorganic materials, our pure organic radical compounds are extremely soluble in the green solvents such as ethanol and DMSO, which make them show excellent reproducibility and solution processibility. ${ }^{69-78}$ However, the high performance photothermal materials are not soluble in any solvents and challenging to reproduce their preparation. As shown in Figure S17 and Table S2, water evaporation efficiency and rate for reported materials were counted, 10, 59, 66-85 where the star-shaped dots represent pure organic materials. In the current high-efficiency pure organic system (CR-TPE-T), the main disadvantages include the following points: expensive raw materials, complex synthesis, difficulty in purification and very low yield. The TPA-TPA- ${ }_{6}$ is cheap and easy to synthesize via only 2 steps with very high yield and convenient purification process. ${ }^{10}$ In addition, our results show that this design concept will produce large space for structural optimization, meanwhile it shows comparable performance with previous pure organic photothermal materials. 10,12,28 In addition, we further verify the feasibility of applying TPA-TPA- ${ }_{6}$ for seawater desalination. Notably, after desalination, the concentrations of all four primary ions $\left(\mathrm{Na}^{+}, \mathrm{Mg}^{2+}, \mathrm{Ca}^{2+}\right.$, and $\mathrm{K}^{+}$) originally present in seawater were significantly reduced (Figure S18), which are lower than the values through the typical membrane-based method. 86 Thus, the stable open-shell radicals showed excellent solar-driven desalination performance and promising practical application potential in future. 11,13,35,87

In summary, with low cost starting raw materials a series of high spin open-shell ANAR molecules were prepared with simple process and exhibit excellent electrochemical, photothermal stability and possess excellent photothermal conversion properties. From the comparison of different molecular structures and performances, we found that TPATPA-O 6 displays wider near-infrared absorption, higher paramagnetic behavior and photothermal conversion efficiency. A high solar thermal conversion efficiency of $89.41 \%$ and water evaporation rate of $1.293 \mathrm{~kg} \mathrm{~m}^{-2} \mathrm{~h}^{-1}$ under 1 sun irradiation are detected and represent as one of the most efficient photothermal conversion materials. And it was found that the intensity of radicals is positively 
A
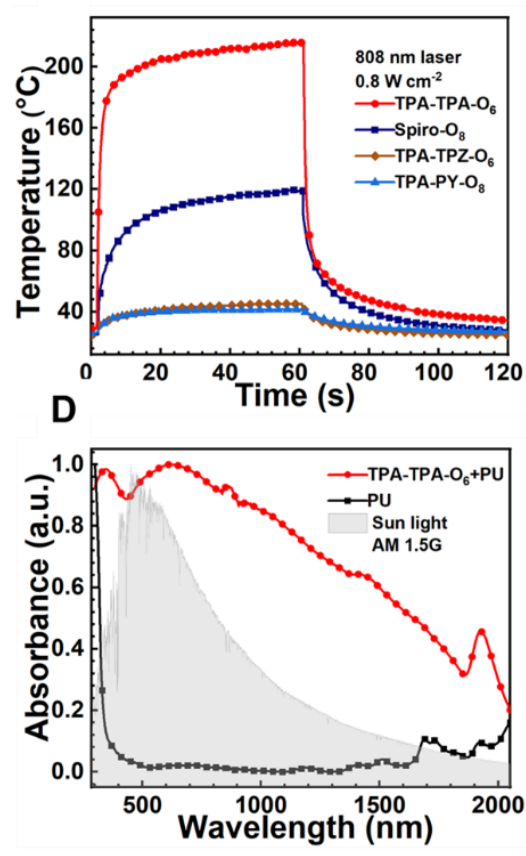

B
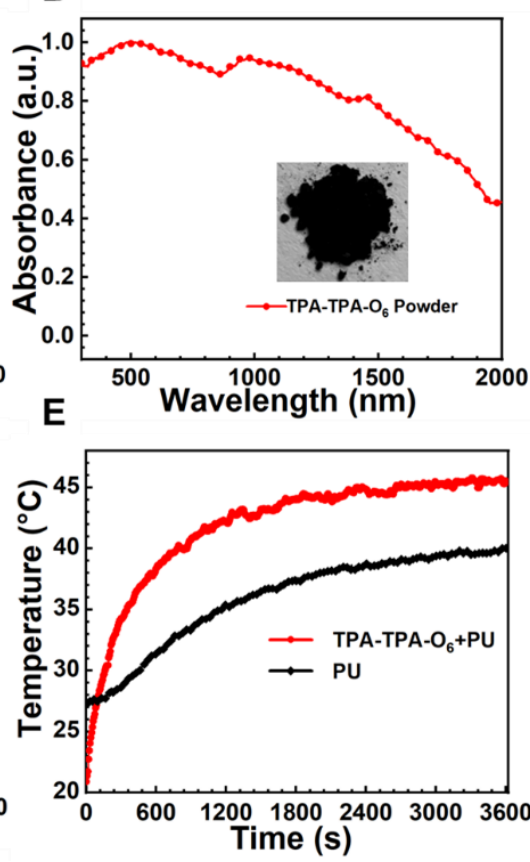

C
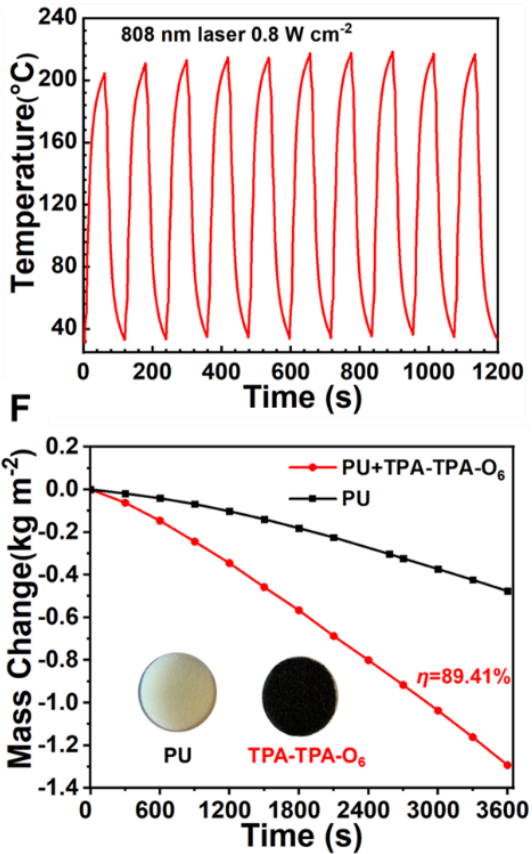

Figure 6. (A) Photothermal conversion behavior of star-shaped radical materials under $808 \mathrm{~nm}$ laser $(0.8 \mathrm{~W} \mathrm{~cm}-2)$ and then turned off in powder. (B) The absorption of TPA-TPA- ${ }_{6}$ in powder. (C) Anti-photobleaching property of TPA-TPA- ${ }_{6}$ powder during ten cycles of heating-cooling processes. (D) The absorption of PU, PU+TPA-TPA- ${ }_{6}$ foams and the solar spectral irradiance (gray). (E) The temperature changes of PU and PU+TPA-TPA- ${ }_{6}$ foams floating on water against sunlight irradiation time. (F) Water evaporation curves with PU foam and PU+TPA-TPA- $\mathrm{O}_{6}$ foam under simulated sunlight with an intensity of $1 \mathrm{~kW} \mathrm{~m}{ }^{-2}$ ( 1 sun) (Insert: Digital photos of pure PU and PU+TPA-TPA- ${ }_{6}$ foams, respectively.)

correlated with the photothermal conversion characteristics. In summary, this study provides a novel strategy for the design of stable high spin radical materials. Furthermore, considering their totally restricted radiation decay and potential high conductivity, we will further develop ANAR materials with enhanced planarity for applications in the fields of photothermal conversion, organic electronic, spintronics as well as magnetic devices.

\section{ASSOCIATED CONTENT}

The Supporting Information is available free of charge at http://pubs.acs.org.

\section{AUTHOR INFORMATION}

\section{Corresponding Author}

Yuan Li - Institute of Polymer Optoelectronic Materials and Devices, State Key Laboratory of Luminescent Materials and Devices, South China University of Technology, Guangzhou 510640, China; orcid.org/0000-0002-7931-9879.

Email: celiy@scut.edu.cn

Authors

Zejun Wang - Institute of Polymer Optoelectronic Materials and Devices, State Key Laboratory of Luminescent Materials and Devices, South China University of Technology, Guangzhou 510640, China Jiawen Zhou - Institute of Polymer Optoelectronic Materials and Devices, State Key Laboratory of Luminescent Materials and Devices, South China University of Technology, Guangzhou 510640, China
Yiheng Zhang - Institute of Polymer Optoelectronic Materials and Devices, State Key Laboratory of Luminescent Materials and Devices, South China University of Technology,

Guangzhou 510640, China

Weiya Zhu - Institute of Polymer Optoelectronic Materials and Devices, State Key Laboratory of Luminescent Materials and Devices, South China University of Technology, Guangzhou 510640, China

\section{Author Contributions}

Y.L. leaded the project and conceived the whole study; Z.J. Wang and J.W. Zhou performed the sample preparation and test; Y.L., Z.J. Wang, J.W. Zhou, Y.W. Zhu and Y.H. Zhang performed the test and data analysis; Y.L., Z.J. Wang and Y.W. Zhu wrote and revised the manuscript. All authors discussed the results and approved the final version of the manuscript.

\section{Funding Sources}

The work was financially supported by the Natural Science Foundation of China (51973063), the Tip-top Scientific and Technical Innovative Youth Talents of Guangdong Special Support Program (2019TQ05C890), the Pearl River S\&T Nova Program of Guangzhou (201710010194) and the Fund of Guangdong Provincial Key Laboratory of Luminescence from Molecular Aggregates (2019B030301003).

\section{Notes}

The authors declare no competing financial interest.

\section{ACKNOWLEDGMENT}


The work was financially supported by the Natural Science Foundation of China (51973063), the Tip-top Scientific and Technical Innovative Youth Talents of Guangdong Special Support Program (2019TQ05C890), the Pearl River S\&T Nova Program of Guangzhou (201710010194) and the Fund of Guangdong Provincial Key Laboratory of Luminescence from Molecular Aggregates (2019B030301003). Thanks to Professor Jia Zhu from Nanjing University for his valuable suggestions on paper writing.

\section{REFERENCES}

1. Tan, Y.; Casetti, N. C.; Boudouris, B. W.; Savoie, B. M. Molecular Design Features for Charge Transport in Nonconjugated Radical Polymers. J Am Chem Soc. 2021, 143, 11994-12002.

2. De Sousa, J. A.; Bejarano, F.; Gutierrez, D.; Leroux, Y. R.; NowikBoltyk, E. M.; Junghoefer, T.; Giangrisostomi, E.; Ovsyannikov, R.; Casu, M. B.; Veciana, J.; Mas-Torrent, M.; Fabre, B.; Rovira, C.; Crivillers, N. Exploiting the versatile alkyne-based chemistry for expanding the applications of a stable triphenylmethyl organic radical on surfaces. Chem Sci. 2020, 11, 516-524.

3. Kimura, S.; Kimura, S.; Kato, K.; Teki, Y.; Nishihara, H.; Kusamoto, T. A ground-state-dominated magnetic field effect on the luminescence of stable organic radicals. Chem Sci. 2021, 12, 2025-2029.

4. Harvey, S. M.; Wasielewski, M. R. Photogenerated SpinCorrelated Radical Pairs: From Photosynthetic Energy Transduction to Quantum Information Science. J Am Chem Soc. 2021, 143, 15508-15529.

5. Kimura, S.; Matsuoka, R.; Kimura, S.; Nishihara, H.; Kusamoto, T. Radical-Based Coordination Polymers as a Platform for Magneto luminescence. J Am Chem Soc. 2021, 143, 5610-5615.

6. Ratera, I.; Vidal-Gancedo, J.; Maspoch, D.; Bromley, S. T.; Crivillers, N.; Mas-Torrent, M. Perspectives for polychlorinated trityl radicals. Journal of Materials Chemistry C. 2021, 9, 1061010623.

7. Nguyen, T. P.; Easley, A. D.; Kang, N.; Khan, S.; Lim, S. M.; Rezenom, Y. H.; Wang, S.; Tran, D. K.; Fan, J.; Letteri, R. A.; He, X.; Su, L.; Yu, C. H.; Lutkenhaus, J. L.; Wooley, K. L. Polypeptide organic radical batteries. Nature. 2021, 593, 61-66.

8. Mahmood, J.; Baek, J.-B. Room-Temperature Organic Ferromagnetism. Chem. 2019, 5, 1012-1014.

9. Liu, Z.; Wu, M.; Lan, M.; Zhang, W. Boosting cancer therapy efficiency via photoinduced radical production. Chem Sci. 2021, 12, 9500-9505.

10. Chen, G.; Sun, J.; Peng, Q.; Sun, Q.; Wang, G.; Cai, Y.; Gu, X.; Shuai, Z.; Tang, B. Z. Biradical-Featured Stable Organic-SmallMolecule Photothermal Materials for Highly Efficient Solar-Driven Water Evaporation. Adv Mater. 2020, 32, 1908537.

11. Chen, Z. X.; Li, Y.; Huang, F. Persistent and Stable Organic Radicals: Design, Synthesis, and Applications. Chem, 2020, 7, 288332.

12. Jung, H. S.; Verwilst, P.; Sharma, A.; Shin, J.; Sessler, J. L.; Kim, J. S. Organic molecule-based photothermal agents: an expanding photothermal therapy universe. Chem Soc Rev. 2018, 47, 22802297.

13. Fuzil, N. S.; Othman, N. H.; Alias, N. H.; Marpani, F.; Othman, M. H. D.; Ismail, A. F.; Lau, W. J.; Li, K.; Kusworo, T. D.; Ichinose, I.; Shirazi, M. M. A. A review on photothermal material and its usage in the development of photothermal membrane for sustainable clean water production. Desalination. 2021, 517, 115259.

14. Joo, Y.; Huang, L.; Eedugurala, N.; London, A. E.; Kumar, A.; Wong, B. M.; Boudouris, B. W.; Azoulay, J. D. Thermoelectric Performance of an Open-Shell Donor-Acceptor Conjugated Polymer Doped with a Radical-Containing Small Molecule. Macromolecules. 2018, 51, 3886-3894.
15. Hu, W.; Prasad, P. N.; Huang, W. Manipulating the Dynamics of Dark Excited States in Organic Materials for Phototheranostics. Acc Chem Res. 2021, 54, 697-706.

16. Dai, X.; Meng, Q.; Zhang, F.; Zou, Y.; Di, C.-a.; Zhu, D. Electronic structure engineering in organic thermoelectric materials. Journal of Energy Chemistry. 2021, 62, 204-219.

17. Tam, T. L. D.; Lin, M.; Handoko, A. D.; Lin, T. T.; Xu, J. Highperformance $\&$ thermally stable n-type polymer thermoelectrics based on a benzyl viologen radical cation-doped ladder-type conjugated polymer. Journal of Materials Chemistry A. 2021, 9, 11787-11793.

18. Zhao, D.; Jiang, Q.; Jia, Y.; Zhou, J.; Zheng, N.; Hu, D.; Ma, Y. High performance n-type thermoelectric material based on naphthalenediimide radical anions. Materials Today Energy. 2021, 21, 100710.

19. Li, T.; Tan, G.; Shao, D.; Li, J.; Zhang, Z.; Song, Y.; Sui, Y.; Chen, S.; Fang, Y.; Wang, X. Magnetic Bistability in a Discrete Organic Radical. J Am Chem Soc. 2016, 138, 10092-10095.

20. Kuwabara, Y.; Kitazawa, M.; Nosaka, A. Y.; Nosaka, Y. Spin Dynamics of the Radicals Produced from Methane Sulfonic Acid and Acetic Acid by Hydroxyl Radicals in Aqueous Solution Studied by Means of Time-Resolved Electron Spin Resonance Spectroscopy. J Phys Chem A. 2021, 125, 1816-1826.

21. Deumal, M.; Vela, S.; Fumanal, M.; Ribas-Arino, J.; Novoa, J. J. Insights into the magnetism and phase transitions of organic radical-based materials. Journal of Materials Chemistry C. 2021, 9 , 10624-10646.

22. Poggini, L.; Cucinotta, G.; Sorace, L.; Caneschi, A.; Gatteschi, D.; Sessoli, R.; Mannini, M. Nitronyl nitroxide radicals at the interface: a hybrid architecture for spintronics. Rendiconti Lincei. Scienze Fisiche e Naturali. 2018, 29, 623-630.

23. Tang, H.; Liu, Z.; Tang, Y.; Du, Z.; Liang, Y.; Hu, Z.; Zhang, K.; Huang, F.; Cao, Y. Organic diradicals enabled N-type self-doped conjugated polyelectrolyte with high transparency and enhanced conductivity. Giant. 2021, 6, 100053.

24. Ji, L.; Shi, J.; Wei, J.; Yu, T.; Huang, W, Air-Stable Organic Radicals: New-Generation Materials for Flexible Electronics? Adv. Mater 2020, 32, 1908015.

25. Hu, X.; Wang, W.; Wang, D.; Zheng, Y. The electronic applications of stable diradicaloids: present and future. Journal of Materials Chemistry C. 2018, 6, 11232-11242.

26. Tang, B.; Zhao, J.; Xu, J.-F.; Zhang, X. Tuning the stability of organic radicals: from covalent approaches to non-covalent approaches. Chemical Science. 2020, 11, 1192-1204.

27. Ratera, I.; Veciana, J. Playing with organic radicals as building blocks for functional molecular materials. Chem Soc Rev. 2012, 41, 303-349.

28. Shi, Y.; Deng, X.; Bao, S.; Liu, B.; Liu, B.; Ma, P.; Cheng, Z.; Pang, M.; Lin, J. Self-Templated Stepwise Synthesis of Monodispersed Nanoscale Metalated Covalent Organic Polymers for In Vivo Bioimaging and Photothermal Therapy. Chem Asian J. 2017, 12, 2183-2188.

29. Mi, Z.; Yang, P.; Wang, R.; Unruangsri, J.; Yang, W.; Wang, C.; Guo, J. Stable Radical Cation-Containing Covalent Organic Frameworks Exhibiting Remarkable Structure-Enhanced Photothermal Conversion. J Am Chem Soc. 2019, 141, 1443314442.

30. Lu, B.; Chen, Y.; Li, P.; Wang, B.; Mullen, K.; Yin, M. Stable radical anions generated from a porous perylenediimide metalorganic framework for boosting near-infrared photothermal conversion. Nat Commun. 2019, 10, 767.

31. Ren, F.; Li, Z.; Li, K.; Zheng, X.; Shi, J.; Zhang, C.; Guo, H.; Tong, B.; Xi, L.; Cai, Z.; Dong, Y. Donor strategy for promoting nonradiative decay to achieve an efficient photothermal therapy for treating cancer. Science China Chemistry. 2021, 64, 1530-1539.

32. Zhao, W.; Gong, H.; Song, Y.; Li, B.; Xu, N.; Min, X.; Liu, G.; Zhu, B.; Zhou, L.; Zhang, X. X.; Zhu, J. Hierarchically Designed Salt Resistant Solar Evaporator Based on Donnan Effect for Stable and 
High - Performance Brine Treatment. Advanced Functional Materials. 2021, 31, 2100025.

33. Li, X.; Li, J.; Lu, J.; Xu, N.; Chen, C.; Min, X.; Zhu, B.; Li, H.; Zhou, L.; Zhu, S.; Zhang, T.; Zhu, J. Enhancement of Interfacial Solar Vapor Generation by Environmental Energy. Joule. 2018, 2, 1331-1338.

34. Wang, F.; Xu, N.; Zhao, W.; Zhou, L.; Zhu, P.; Wang, X.; Zhu, B.; Zhu, J. A high-performing single-stage invert-structured solar water purifier through enhanced absorption and condensation. Joule. 2021, 5, 1602-1612.

35. Razaqpur, A. G.; Wang, Y.; Liao, X.; Liao, Y.; Wang, R. Progress of photothermal membrane distillation for decentralized desalination: A review. Water Res. 2021, 201, 117299.

36. Li, K.; Gao, M.; Li, Z.; Yang, H.; Jing, L.; Tian, X.; Li, Y.; Li, S.; Li, H.; Wang, Q.; Ho, J. S.; Ho, G. W.; Chen, P.-Y. Multi-interface engineering of solar evaporation devices via scalable, synchronous thermal shrinkage and foaming. Nano Energy. 2020, 74, 104875.

37. Burke, D. J.; Lipomi, D. J. Green chemistry for organic solar cells. Energy \& Environmental Science. 2013, 6, 2053-2066.

38. Chen, H.; Shao, L.; Ming, T.; Sun, Z.; Zhao, C.; Yang, B.; Wang, J. Understanding the photothermal conversion efficiency of gold nanocrystals. Small. 2010, 6, 2272-2280.

39. Ma, S.; Chiu, C. P.; Zhu, Y.; Tang, C. Y.; Long, H.; Qarony, W.; Zhao, X.; Zhang, X.; Lo, W. H.; Tsang, Y. H. Recycled waste black polyurethane sponges for solar vapor generation and distillation. Applied Energy. 2017, 206, 63-69.

40. Wang, D.; Zhang, Z.; Lin, L.; Liu, F.; Wang, Y.; Guo, Z.; Li, Y.; Tian, H.; Chen, X. Porphyrin-based covalent organic framework nanoparticles for photoacoustic imaging-guided photodynamic and photothermal combination cancer therapy. Biomaterials. 2019, 223, 119459.

41. Chen, C.; Liang, Q.; Chen, Z.; Zhu, W.; Wang, Z.; Li, Y.; Xiong, X. Phenoxy-Radical-induced Formation of Dual-Layered Protection Film for High-Rate and Dendrite-free Lithium Metal Anodes. Angew Chem Int Ed Engl. 2021, 10.1002/anie.202110441.

42. Zhang, X.; Li, Y.; Chen, Z.; Li, P.; Chen, R.; Peng, X. Molecular engineering of narrow bandgap porphyrin derivatives for highly efficient photothermal conversion. Dyes and Pigments. 2021, 192, 109460.

43. Olankitwanit, A.; Rajca, S.; Rajca, A. Aza-m-Xylylene Diradical with Increased Steric Protection of the Aminyl Radicals. J Org Chem. 2015, 80, 5035-5044.

44. Rudebusch, G. E.; Zafra, J. L.; Jorner, K.; Fukuda, K.; Marshall, J. L.; Arrechea-Marcos, I.; Espejo, G. L.; Ponce Ortiz, R.; GomezGarcia, C. J.; Zakharov, L. N.; Nakano, M.; Ottosson, H.; Casado, J.; Haley, M. M. Diindeno-fusion of an anthracene as a design strategy for stable organic biradicals. Nat Chem. 2016, 8, 753-759.

45. Gallagher, N. M.; Bauer, J. J.; Pink, M.; Rajca, S.; Rajca, A. HighSpin Organic Diradical with Robust Stability. J Am Chem Soc. 2016 138, 9377-9380.

46. Lu, Z.; Quanz, H.; Burghaus, O.; Hofmann, J.; Logemann, C.; Beeck, S.; Schreiner, P. R.; Wegner, H. A. Stable Organic Neutral Diradical via Reversible Coordination. J Am Chem Soc. 2017, 139, 18488-18491.

47. Xue, Y.; Guo, P.; Yip, H.-L.; Li, Y.; Cao, Y. General design of selfdoped small molecules as efficient hole extraction materials for polymer solar cells. Journal of Materials Chemistry A. 2017, 5, 3780-3785.

48. Li, Y.; Li, L.; Wu, Y.; Li, Y. A Review on the Origin of Synthetic Metal Radical: Singlet Open-Shell Radical Ground State? The Journal of Physical Chemistry C. 2017, 121, 8579-8588.

49. Zhou, J.; Zhu, W.; Zeng, M.; Yang, Q.; Li, P.; Lan, L.; Peng, J.; Li, Y.; Huang, F.; Cao, Y. Aromatic inorganic acid radical. Science China Chemistry. 2019, 62, 1656-1665.

50. Chen, Z.; Li, W.; Zhang, Y.; Wang, Z.; Zhu, W.; Zeng, M.; Li, Y. Aggregation-Induced Radical of Donor-Acceptor Organic Semiconductors. The Journal of Physical Chemistry Letters. 2021, doi.org/10.1021/acs.jpclett.1c02463.
51. Waked, A. E.; Ostadsharif Memar, R.; Stephan, D. W. NitrogenBased Lewis Acids Derived from Phosphonium Diazo Cations. Angew Chem Int Ed Engl. 2018, 57, 11934-11938.

52. Wang, J.; Kim, G.; Sandoval-Salinas, M. E.; Phan, H.; Gopalakrishna, T. Y.; Lu, X.; Casanova, D.; Kim, D.; Wu, J. Stable 2D anti-ferromagnetically coupled fluorenyl radical dendrons. Chem Sci. 2018, 9, 3395-3400.

53. Shahrivarkevishahi, A.; Luzuriaga, M. A.; Herbert, F. C.; Tumac, A. C.; Brohlin, O. R.; Wijesundara, Y. H.; Adlooru, A. V.; Benjamin, C.; Parsamian, P.; Gadhvi, J.; De Nisco, N. J.; Gassensmith, J. J. PhotothermalPhage: A Virus-Based Photothermal Therapeutic Agent. J Am Chem Soc. 2021, doi.org/10.1021/jacs.1c05090.

54. Cui, B. B.; Zhu, C.; Yang, S.; Han, Y.; Yang, N.; Zhang, L.; Wang, Y.; Jia, Y.; Zhao, L.; Chen, Q. Two Low-Cost and Efficient HoleTransporting Materials for n-i-p Type Organic-Inorganic Hybrid Perovskite Solar Cells. ACS Omega. 2018, 3, 10791-10797.

55. Choi, H.; Paek, S.; Lim, N.; Lee, Y. H.; Nazeeruddin, M. K.; Ko, J. Efficient perovskite solar cells with $13.63 \%$ efficiency based on planar triphenylamine hole conductors. Chemistry. 2014, 20, 10894-10899.

56. Su, J.; Xu, N.; Murase, R.; Yang, Z. M.; D'Alessandro, D. M.; Zuo, J. L.; Zhu, J. Persistent Radical Tetrathiafulvalene-Based 2D MetalOrganic Frameworks and Their Application in Efficient Photothermal Conversion. Angew Chem Int Ed Engl. 2021, 60, 4789-4795.

57. Li, Y.; Heng, W. K.; Lee, B. S.; Aratani, N.; Zafra, J. L.; Bao, N.; Lee, R.; Sung, Y. M.; Sun, Z.; Huang, K. W.; Webster, R. D.; Lopez Navarrete, J. T.; Kim, D.; Osuka, A.; Casado, J.; Ding, J.; Wu, J. Kinetically blocked stable heptazethrene and octazethrene: closedshell or open-shell in the ground state? J Am Chem Soc. 2012, 134, 14913-14922.

58. Abe, M. Diradicals. Chem Rev. 2013, 113, 7011-88.

59. Ma, S.; Qarony, W.; Hossain, M. I.; Yip, C. T.; Tsang, Y. H. Metalorganic framework derived porous carbon of light trapping structures for efficient solar steam generation. Solar Energy Materials and Solar Cells. 2019, 196, 36-42.

60. Wang, Y.; Zhu, W.; Du, W.; Liu, X.; Zhang, X.; Dong, H.; Hu, W. Cocrystals Strategy towards Materials for Near-Infrared Photothermal Conversion and Imaging. Angew Chem Int Ed Engl. 2018, 57, 3963-3967.

61. Wang, D.; Kan, X.; Wu, C.; Gong, Y.; Guo, G.; Liang, T.; Wang, L.; Li, Z.; Zhao, Y. Charge transfer co-crystals based on donor-acceptor interactions for near-infrared photothermal conversion. Chem Commun. 2020, 56, 5223-5226.

62. Cao, Y.; Dou, J.-H.; Zhao, N.-j.; Zhang, S.; Zheng, Y.-Q.; Zhang, J.-P.; Wang, J.-Y.; Pei, J.; Wang, Y. Highly Efficient NIR-II Photothermal Conversion Based on an Organic Conjugated Polymer. Chemistry of Materials. 2016, 29, 718-725.

63. Yang, M. Q.; Tan, C. F.; Lu, W.; Zeng, K.; Ho, G. W. Spectrum Tailored Defective 2D Semiconductor Nanosheets Aerogel for Full - Spectrum - Driven Photothermal Water Evaporation and Photochemical Degradation. Advanced Functional Materials. 2020, 30, 2004460.

64. Saleque, A. M.; Ma, S.; Ahmed, S.; Hossain, M. I.; Qarony, W.; Tsang, Y. H. Solar Driven Interfacial Steam Generation Derived from Biodegradable Luffa Sponge. Advanced Sustainable Systems. 2021, 5, 2000291.

65. Nawaz, F.; Yang, Y.; Zhao, S.; Sheng, M.; Pan, C.; Que, W. Innovative salt-blocking technologies of photothermal materials in solar-driven interfacial desalination. Journal of Materials Chemistry A. 2021, 9, 16233-16254.

66. Li, Y.; Gao, T.; Yang, Z.; Chen, C.; Luo, W.; Song, J.; Hitz, E.; Jia, C.; Zhou, Y.; Liu, B.; Yang, B.; Hu, L. 3D-Printed, All-in-One Evaporator for High-Efficiency Solar Steam Generation under 1 Sun Illumination. Adv Mater. 2017, 29, 1700981.

67. Zhu, L.; Ding, T.; Gao, M.; Peh, C. K. N.; Ho, G. W. Shape Conformal and Thermal Insulative Organic Solar Absorber Sponge for Photothermal Water Evaporation and Thermoelectric Power Generation. Advanced Energy Materials. 2019, 9, 1900250. 
68. Zou, Y.; Chen, X.; Yang, P.; Liang, G.; Yang, Y.; Gu, Z.; Li, Y. Regulating the absorption spectrum of polydopamine. Science Advances. 2020, 6, eabb4696.

69. Yang, Y.; Yang, X.; Fu, L.; Zou, M.; Cao, A.; Du, Y.; Yuan, Q.; Yan, C.-H. Two-Dimensional Flexible Bilayer Janus Membrane for Advanced Photothermal Water Desalination. ACS Energy Letters. 2018, 3, 1165-1171.

70. Hu, X.; Xu, W.; Zhou, L.; Tan, Y.; Wang, Y.; Zhu, S.; Zhu, J. Tailoring Graphene Oxide-Based Aerogels for Efficient Solar Steam Generation under One Sun. Adv Mater. 2017, 29, 1604031.

71. Xu, N.; Hu, X.; Xu, W.; Li, X.; Zhou, L.; Zhu, S.; Zhu, J. Mushrooms as Efficient Solar Steam-Generation Devices. Adv Mater. 2017, 29, 1606762.

72. Zhang, L.; Tang, B.; Wu, J.; Li, R.; Wang, P. Hydrophobic Lightto-Heat Conversion Membranes with Self-Healing Ability for Interfacial Solar Heating. Adv Mater. 2015, 27, 4889-4894.

73. Gao, M.; Peh, C. K.; Phan, H. T.; Zhu, L.; Ho, G. W. Solar Absorber Gel: Localized Macro-Nano Heat Channeling for Efficient Plasmonic $\mathrm{Au}$ Nanoflowers Photothermic Vaporization and Triboelectric Generation. Advanced Energy Materials. 2018, 8, 1800711.

74. Li, K.; Chang, T. H.; Li, Z.; Yang, H.; Fu, F.; Li, T.; Ho, J. S.; Chen, P. Y. Biomimetic MXene Textures with Enhanced Light - to - Heat Conversion for Solar Steam Generation and Wearable Thermal Management. Advanced Energy Materials. 2019, 9, 1901687.

75. Li, W.; Tekell, M. C.; Huang, Y.; Bertelsmann, K.; Lau, M.; Fan, D. Synergistic High-Rate Solar Steaming and Mercury Removal with $\mathrm{MoS}_{2} / \mathrm{C} @$ Polyurethane Composite Sponges. Advanced Energy Materials. 2018, 8, 1802108.

76. Liu, H.; Chen, C.; Chen, G.; Kuang, Y.; Zhao, X.; Song, J.; Jia, C.; Xu, X.; Hitz, E.; Xie, H.; Wang, S.; Jiang, F.; Li, T.; Li, Y.; Gong, A.; Yang R.; Das, S.; Hu, L. High-Performance Solar Steam Device with Layered Channels: Artificial Tree with a Reversed Design. Advanced Energy Materials. 2018, 8, 1701616.

77. Ye, M.; Jia, J.; Wu, Z.; Qian, C.; Chen, R.; O'Brien, P. G.; Sun, W.; Dong, Y.; Ozin, G. A. Synthesis of Black $\mathrm{TiO}_{\mathrm{x}}$ Nanoparticles by $\mathrm{Mg}$ Reduction of $\mathrm{TiO}_{2}$ Nanocrystals and their Application for Solar Water Evaporation. Advanced Energy Materials. 2017, 7, 1601811.

78. Shi, Y.; Li, R.; Shi, L.; Ahmed, E.; Jin, Y.; Wang, P. A Robust $\mathrm{CuCr}_{2} \mathrm{O}_{4} / \mathrm{SiO}_{2}$ Composite Photothermal Material with Underwater
Black Property and Extremely High Thermal Stability for SolarDriven Water Evaporation. Advanced Sustainable Systems. 2018, 2, 1700145 .

79. Wang, F.; Su, Y.; Li, Y.; Wei, D.; Sun, H.; Zhu, Z.; Liang, W.; Li, A. Salt-Resistant Photothermal Materials Based on Monolithic Porous Ionic Polymers for Efficient Solar Steam Generation. ACS Applied Energy Materials. 2020, 3, 8746-8754.

80. Zhang, P.; Li, J.; Lv, L.; Zhao, Y.; Qu, L. Vertically Aligned Graphene Sheets Membrane for Highly Efficient Solar Thermal Generation of Clean Water. ACS Nano 2017, 20, 5087.

81. Liu, F.; Zhao, B.; Wu, W.; Yang, H.; Ning, Y.; Lai, Y.; Bradley, R. Low Cost, Robust, Environmentally Friendly GeopolymerMesoporous Carbon Composites for Efficient Solar Powered Steam Generation. Advanced Functional Materials. 2018, 28, 1803266.

82. Liu, Z.; Wu, B.; Zhu, B.; Chen, Z.; Zhu, M.; Liu, X. Continuously Producing Water steam and Concentrated Brine from Seawater by Hanging Photothermal Fabrics under Sunlight. Advanced Functional Materials. 2019, 29, 1905485.

83. Ming, X.; Guo, A.; Zhang, Q.; Guo, Z.; Yu, F.; Hou, B.; Wang, Y.; Homewood, K. P.; Wang, X. 3D macroscopic graphene oxide/MXene architectures for multifunctional water purification. Carbon. 2020, 167, 285-295.

84. He, S.; Chen, C.; Kuang, Y.; Mi, R.; Liu, Y.; Pei, Y.; Kong, W.; Gan, W.; Xie, H.; Hitz, E.; Jia, C.; Chen, X.; Gong, A.; Liao, J.; Li, J.; Ren, Z. J.; Yang, B.; Das, S.; Hu, L. Nature-inspired salt resistant bimodal porous solar evaporator for efficient and stable water desalination. Energy \& Environmental Science. 2019, 12, 1558-1567.

85. Chen, C.; Zhou, L.; Yu, J.; Wang, Y.; Nie, S.; Zhu, S.; Zhu, J. Dual functional asymmetric plasmonic structures for solar water purification and pollution detection. Nano Energy. 2018, 51, 451 456.

86. Zhou, L.; Tan, Y.; Wang, J.; Xu, W.; Yuan, Y.; Cai, W.; Zhu, S.; Zhu, J. 3D self-assembly of aluminium nanoparticles for plasmonenhanced solar desalination. Nature Photonics. 2016, 10, 393-398.

87. Guo, B.; Huang, Z.; Shi, Q.; Middha, E.; Xu, S.; Liu, B. Organic small molecule based photothermal agents with molecular rotors for malignant breast cancer therapy. Advanced Functional Materials, 2020, 30, 1907093. 
Table of Contents

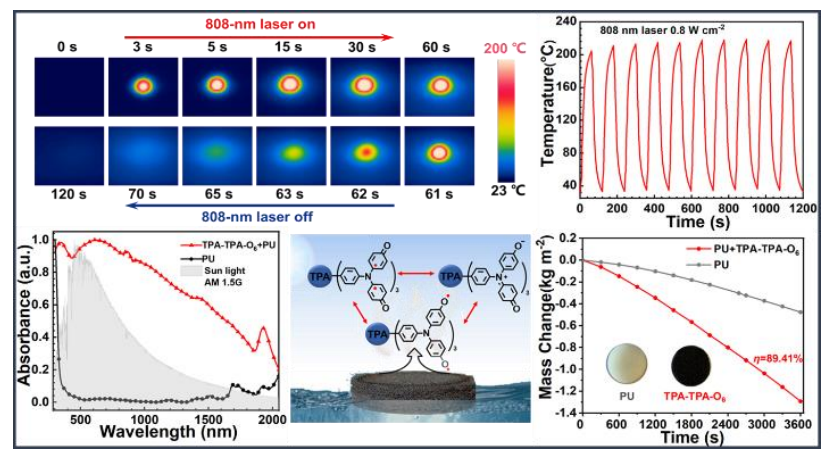

